\begin{tabular}{|c|l|}
\hline Title & The Rim101 pathway contributes to ER stress adaptation through sensing the state of plasma membrane \\
\hline Author(s) & Obara, Keisuke; Kihara, A kio \\
\hline Citation & $\begin{array}{l}\text { Biochemical journal, 474,51-63 } \\
\text { https://doi.org/40.1042/BCJ20160580 }\end{array}$ \\
\hline Issue Date & 2017-01-01 \\
\hline Doc URL & http://hdl.handle.net/2115/68018 \\
\hline Type & article (author version) \\
\hline File Information & kiharabj474.pdf \\
\hline
\end{tabular}

Instructions for use 


\title{
The Rim101 pathway contributes to ER stress adaptation through sensing the state of plasma membrane
}

\author{
Keisuke Obara and Akio Kihara \\ Faculty of Pharmaceutical Sciences, Hokkaido University, Kita 12-jo, Nishi 6-chome, \\ Kita-ku, Sapporo 060-0812, Japan
}

Corresponding author: Akio Kihara

Mailing address: Faculty of Pharmaceutical Sciences, Hokkaido University, Kita 12-jo, Nishi 6-chome, Kita-ku, Sapporo 060-0812, Japan

Tel: +81-11-706-3754

Fax: +81-11-706-4900

E-mail: kihara@pharm.hokudai.ac.jp

Short Title: Adaptation to ER stress through sensing the state of plasma membrane

Key words: ER stress, plasma membrane, contact site, lipid asymmetry, Rim101 pathway, Saccharomyces cerevisiae

\begin{abstract}
Abbreviation list: DMSO, dimethyl sulfoxide; ER, endoplasmic reticulum; ESCRT, endosomal sorting complex required for transport; PM, plasma membrane; PtdSer, phosphatidylserine; Rim21C, C-terminal cytosolic region of Rim21; WT, wild-type
\end{abstract}




\section{Summary statement}

The sensor protein for plasma membrane lipid asymmetry, Rim21, detects ER stress by sensing alterations in the state of the plasma membrane, and triggers an adaptation response via the Rim101 pathway. 


\begin{abstract}
Yeast cells sense alterations in the plasma membrane (PM) lipid asymmetry and external alkalization by the sensor protein Rim21, which functions in the Rim101 pathway. Rim101 signaling is initiated at the PM by the recruitment of the Rim101 signaling complex. The PM physically associates with the cortical endoplasmic reticulum (ER) to form ER-PM contact sites, where several signaling events, lipid exchange, and ion transport take place. In the present study, we investigated the spatial relationship between ER-PM contact sites and the sites of Rim101 signaling. Rim101 signaling mostly proceeds outside ER-PM contact sites in the PM and did not require intact ER-PM contact for its activation. Rather, the Rim101 pathway was constitutively activated by ER-PM contact site disruption, which is known to cause ER stress. ER stress induced by tunicamycin treatment activated the Rim101 pathway. Furthermore, the sensitivity of cells to tunicamycin without ER-PM contact was considerably elevated by the deletion of RIM21. These results suggest that the Rim101 pathway is important for the adaptation to ER stress by compensating for alternations in PM lipid asymmetry induced by ER stress.
\end{abstract}




\section{INTRODUCTION}

Living organisms must respond appropriately to environmental changes. For instance, fungi that grow over a wide $\mathrm{pH}$ range, such as Saccharomyces cerevisiae, Candida albicans, and Aspergillus nidulans, adapt to alkaline conditions via the Rim101 pathway [1-3]. For adaptation to external alkalization, these fungi activate the Rim101 pathway to induce alkaline-responsive genes, which encode for secreted enzymes, cell wall proteins, and proteins required for ion homeostasis [4-7]. For pathogenic fungi, adaptation to external $\mathrm{pH}$ is critical for growth and pathogenicity in the host organisms [2, 3]. Therefore, understanding the mechanism of this adaptation is an important aspect of drug development and agriculture, as well as basic biology.

In the lipid bilayer of the eukaryotic plasma membrane (PM), lipid composition differs between the inner (cytoplasmic) and the outer (extracellular) leaflets, and this difference is called lipid asymmetry [8, 9]. For example, phosphatidylserine (PtdSer), phosphatidylethanolamine, and phosphatidylinositol are mostly confined to the inner leaflet, while phosphatidylcholine and sphingolipids are enriched in the outer leaflet. Maintenance of lipid asymmetry is important for various cellular processes, including generation of membrane potential, establishment of cell polarity, and vesicular transport, whereas transient or irreversible disruption of the lipid asymmetry induces cytokinesis, blood coagulation, and removal of apoptotic cells [10-16]. We previously reported that, in addition to external alkalization, alterations in lipid asymmetry activate the Rim101 pathway and induce the expression of the putative lipid floppases/transporters RSB1 and OPT2 [17-20]; hence, this process may be important for the recovery of functional 
asymmetry. Although the exact molecular mechanism for how the distinct environmental changes, such as altered lipid asymmetry or external alkalization, activate a common Rim101 pathway remains unclear, it is possible that both changes are recognized by the same signal. For example, external alkalization may induce lipid asymmetry changes. In the Rim101 pathway, the PM protein Rim21 acts as a sensor and may recognize altered lipid composition of the inner leaflet of the PM [21, 22]. Upon activation of Rim21, downstream molecules, including the arrestin-related protein Rim8, endosomal sorting complex required for transport (ESCRT) proteins, the Bro1 family protein Rim20, and the calpain-like protein Rim13, are recruited to the PM and form a complex, followed by the activation of the transcription factor Rim101 by proteolytic processing [23-25]. Ubiquitination of certain Rim protein(s) by the ubiquitin ligase Rsp5 seems to be involved in the recruitment of the Rim101 signaling complex to the PM [25].

A considerable portion of the cortical endoplasmic reticulum (ER) is tightly apposed to the PM with an average distance of $33 \mathrm{~nm}[26,27]$. Ribosomes are excluded from the face of the cortical ER adjacent to the PM, and the tethering proteins Tcb1, Tcb2, Tcb3, Scs2, Scs22, and Ist2 physically bridge the ER and the PM [28], forming the ER-PM contact sites. When the genes encoding the six tethering proteins were deleted ( $\Delta$ tether mutant), the ER-PM contacts are disrupted [28]. Recent studies have elucidated that phosphatidylinositol 4-phosphate signaling occurs at ER-PM contact sites [29]. In addition, ER-PM contact sites serve as platforms for the exchange of lipids 
between the ER and the PM, and for ion transport between the ER lumen and the extracellular space via the narrow cytosolic space [30].

In the present study, we investigated the association between ER-PM contact sites and Rim101 signaling. We found that Rim101 signaling occurred mostly outside ER-PM contact sites and was constitutively active in the $\Delta$ tether mutant. ER stress is known to be induced by loss of ER-PM contact sites [28]. The Rim101 pathway was activated by tunicamycin-induced ER stress and was needed for growth under the stress conditions. Thus, the Rim101 pathway is important for the adaptation process in response to ER stress. 


\section{MATERIALS AND METHODS}

The yeast Saccharomyces cerevisiae strains used in this study are listed in Table I. Yeast cells were grown in YPD (1\% yeast extract, 2\% bacto peptone, and 2\% D-glucose) or synthetic complete medium (2\% D-glucose and $0.67 \%$ yeast nitrogen base without amino acids with appropriate supplements) at $30^{\circ} \mathrm{C}$. For alkaline treatment, $100 \mathrm{mM}$ Tris-HCl ( $\mathrm{pH}$ 8.0) was added to the culture medium. A $10 \mathrm{mg} / \mathrm{mL}$ stock solution of tunicamycin (Merck, Darmstadt, Germany) and a $2 \mathrm{mg} / \mathrm{mL}$ stock solution of aureobasidin A (Takara Bio, Shiga, Japan) were prepared in dimethyl sulfoxide (DMSO) and ethanol, respectively.

\section{Genetic manipulation and plasmids}

Gene disruption was conducted by replacing the entire coding region of the gene with a marker gene by homologous recombination. Chromosome fusions of GFP, mCherry, or mRFP to the 3'-terminus of the gene were performed using PCR-based gene disruption and modification as described previously [31]. Briefly, the DNA sequence containing the fluorescent protein-encoding gene, the $A D H 1$ terminator, and a marker was amplified by PCR from the pFA6a vector series [31] with a primer set containing the homologous region of each gene. The amplified fragments were inserted directly into the chromosome by homologous recombination. Integration of $\mathrm{P}_{A D H^{-}} G F P-R I M 21 C$ to the yeast chromosome was performed as described previously [22]. Successful deletions of the genes and tagging were confirmed by genomic PCR and/or fluorescence microscopy. The $\Delta$ tether strain (ANDY198) [28] was kindly provided by Dr. C. J. 
Stefan (Medical Research Council, UK). The pFI1 plasmid encoding HA-RIM101 was given to us by Dr. T. Maeda (University of Tokyo, Japan). The pMO13 plasmid, which encodes KAR2 (1-135)-GFP-HDEL for the visualization of the ER, was a gift from Dr. T. Yoko-o (National Institute of Advanced Industrial Science and Technology, Japan).

\section{Immunoblot analysis}

Total cell lysates were prepared by the alkaline/trichloroacetic acid method and subjected to immunoblot analyses using Immobilon $^{\mathrm{TM}}$ polyvinylidene difluoride membrane (Millipore, Billerica, MA) as described previously [32]. Anti-HA TANA2 (0.5 $\mu \mathrm{g} / \mathrm{ml}$; Medical \& Biological Laboratories, Nagoya, Japan) or anti-Pgk1 (0.5 $\mu \mathrm{g} / \mathrm{ml}$; Thermo Fisher Scientific, Waltham, USA) antibodies were used as the primary antibody, and HRP-conjugated anti-mouse IgG $\mathrm{F}(\mathrm{ab})_{2}$ fragment (1:7500 dilution; GE Healthcare Life Sciences, Little Chalfont, UK) was used as the secondary antibody. Immunodetection was performed using the Western Lightning ECL Pro system (PerkinElmer Life Sciences, Waltham, MA, USA) with an X-ray film.

\section{Microscopy}

Fluorescence images were captured using a fluorescence microscope (DM5000B, Leica Microsystems, Wetzlar, Germany) equipped with a cooled CCD camera (DFC365FX, Leica Microsystems), under the control of LAS AF software (version 2.60, Leica Microsystems). In some cases, contrast of the images was enhanced by a linear adjustment using Photoshop CS3 software (Adobe; San Jose, CA, USA). 


\section{RESULTS}

\section{Rim101 signaling proceeds outside ER-PM contact sites in the PM}

The signaling events of the Rim101 pathway proceed at the PM by recruiting the Rim101 signaling complex, which includes the ESCRT-III subunit Snf7 and the Bro1 family protein Rim20 (Fig. 1A and B) [23, 25]. Since a considerable part of the cortical ER associates with the PM, we first investigated the spatial relationship between the ER-PM contact sites and the locations where Rim101 signaling complexes form. To this end, the signaling complex and ER-PM contact sites were visualized by chromosomally-expressed Rim20-GFP and mRFP-tagged Tcb1, one of the tethering protein bridging the ER and PM, respectively. In response to external alkalization, Rim20 accumulated at the PM [25] (Fig. 1C). Most of the Rim20 puncta at the PM did not merge with the Tcb1 signal, and were often observed between the Tcb1 signals. This localization pattern was more evident when the focal plane was adjusted to the cell periphery. We also visualized the entirety of the ER using mCherry-tagged Sec61, an integral ER membrane protein. Again, most of Rim20 puncta did not colocalize with the Sec61 signal (Fig. 1D). Only 6.8\% and 7.1\% of Rim20 puncta at the PM merged with the Tcb1 and Sec61 signals, respectively (Fig. 1E). Snf7 is another subunit of the Rim101 signaling complex. Although Snf7 usually localizes to the endosome, a fraction of Snf7 is recruited to the PM when the Rim101 pathway is activated [25]. Furthermore, Vps27, an ESCRT-0 component, is required for localization of Snf7 to the endosome but not to the PM; hence, Snf7 signal is mostly detected at the PM in vps27 $\Delta$ cells after external alkalization [25]. We analyzed the spatial relationship between Snf7 puncta at 
the PM and ER-PM contact sites in alkali-treated vps27A cells. As with Rim20, the majority of the Snf7 puncta did not merge with the ER-PM contact site marker Tcb1 (Fig. 1F). These observations indicate that the Rim101 signaling complex is mainly formed at sites where the ER is not physically attaching to the PM, although we do not completely exclude the possibility that some of the Rim101 signaling complexes may be formed at contact sites.

To further confirm the localization of Rim101 signaling complex outside ER-PM contact sites, we employed the $\Delta$ tether mutant, in which ER-PM contact sites are disrupted [28]. Indeed, the cortical ER was observed only in the interior of the $\Delta$ tether cells both before and after external alkalization (Fig. 2A). Rim20 still accumulated at the PM in $\Delta$ tether cells upon external alkalization, and this accumulation was dependent on Rim21 (Fig. 2B). Both the fluorescence intensity of Rim20-GFP of whole cells and the intensity associated with the PM puncta were similar between wild-type (WT) and $\Delta$ tether cells (Fig. 2C and D). Snf7 also accumulated at the PM in addition to in the late endosome in alkali-treated $\Delta$ tether cells (Fig. 2E). The fluorescence intensity of Snf7-mRFP of whole cells was slightly higher in $\Delta$ tether cells than in WT cells for unknown reasons (Fig. 2F). These observations indicate that ER-PM contact is not essential for the formation of the Rim101 signaling complex at the PM.

\section{Disruption of ER-PM contact causes constitutive activation of the Rim101 pathway}

During the observations of the Rim101 signaling complex, we found that the signaling complex formed more frequently in $\Delta$ tether cells than in WT cells under normal 
conditions (without external alkalization). The number of Rim20 puncta at the PM was significantly increased in $\Delta$ tether cells (Fig. 3A and B). Likewise, Snf7 puncta at the PM were more easily detected in $\Delta$ tether cells than in WT cells under normal conditions (Fig. 3C). Importantly, Rim20 punctum formation in $\Delta$ tether cells was dependent on the sensor protein Rim21 (Fig. 3A), indicating that the Rim101 signaling complex formation without ER-PM contact sites is an ordinary process triggered by Rim21, rather than an aberrant event that no longer needs Rim21. Considering that ER-PM contact sites function in lipid exchange between the cytosolic leaflet of ER and the inner leaflet of the PM, it is possible that loss of ER-PM contact sites causes a change in lipid composition of the inner leaflet of the PM, leading to altered lipid asymmetry in the PM and activation of the Rim101 pathway.

We recently reported that the C-terminal cytosolic region of Rim21 (Rim21C) senses altered lipid asymmetry, probably through the recognition of altered lipid composition in the inner leaflet of the PM [22]. In that previous study, we showed that GFP-Rim21C was associated with the PM and the yeast bud neck, where lipid asymmetry is altered locally [13] in WT cells under normal conditions, whereas it dissociated from the PM upon activation of the Rim101 pathway (under alkali conditions or in cells with mutations in lipid translocases) [22] (see also Fig. 3D). Thus, the behavior of GFP-Rim21C can be used as an indicator of activation of the Rim101 pathway. Here, we found that GFP-Rim21C was dissociated from the PM in $\Delta$ tether cells under normal conditions (Fig. 3D), indicating constitutive activation of the Rim101 pathway. 
At the final step of Rim101 signaling, the transcription factor Rim101 is activated by proteolytic processing [1], which can be monitored by immunoblotting for the processed Rim101. Processed Rim101 increased in non-treated $\Delta$ tether cells compared with WT cells (Fig. 3E). The processing of Rim101 in $\Delta$ tether cells was completely dependent on Rim21. All of these corroborating results indicate that loss of ER-PM contact leads to the activation of the Rim101 pathway through the Rim21 sensor protein.

We next examined the alkaline-sensitivity of $\Delta$ tether cells. Consistent with a previous report [19], rim21 $\Delta$ cells were sensitive to an alkaline pH (Fig. 3F). Similarly, $\Delta$ tether cells showed a growth defect on alkaline plates, indicating that ER-PM contact is important for alkaline tolerance. Introduction of the $\Delta$ tether mutations into rim21 $\Delta$ cells exacerbated the alkaline sensitivity. This additive effect suggests that the alkaline sensitivity caused by $\Delta$ tether mutations is independent of the Rim101 pathway, at least partly.

\section{Loss of Osh6/7-mediated PtdSer transfer does not cause constitutive activation of the Rim101 pathway}

Since Rim101 signaling was constitutively activated in $\Delta$ tether cells in a Rim21-dependent manner (Fig. 3), we speculated that Rim21 senses alterations in the state of the PM (i.e., altered lipid composition of the inner leaflet) caused by the loss of ER-PM contact. We focused on PtdSer because the Rim101 pathway was activated in a mutant defective in PtdSer synthesis [21]. Firstly, intracellular distribution of PtdSer 
was investigated using a PtdSer reporter protein, GFP-Lact-C2 [33]. GFP-Lact-C2 was mostly detected at the PM in both WT and $\Delta$ tether cells (Fig. 4A). However, in $\Delta$ tether cells a fraction of GFP-Lact-C2 was detected at intracellular membrane structures as well. This localization pattern resembled that of the mutant cells with OSH6 and OSH7 deletions, which mediate non-vesicular, direct transport of PtdSer from the ER to the PM via ER-PM contact sites [34].

We next investigated whether the constitutive activation of the Rim101 pathway in $\Delta$ tether cells was due to loss of Osh6/7-mediated PtdSer transport via ER-PM contact sites. To this end, the processing of Rim101 was monitored in osh6 $\Delta$ osh7 $\Delta$ double mutant cells. We found that the processing of Rim101 was not facilitated in the osh6 4 osh7 $\Delta$ cells (Fig. 4B). Therefore, the activation of the Rim101 pathway in $\Delta$ tether cells cannot be explained solely by loss of Osh6/7-mediated transport of PtdSer.

\section{The Rim101 pathway confers resistance to ER stress}

It is known that the loss of ER-PM contact sites causes ER stress, and therefore $\Delta$ tether cells are hypersensitive to an additional ER stress inducer [28]. We examined the possibility that constitutive activation of the Rim101 pathway in $\Delta$ tether cells was due to ER stress. The Rim101 pathway was stimulated by treatment with tunicamycin, which causes ER stress by inhibiting $N$-linked glycosylation (Fig. 5A). This tunicamycin-induced activation of the Rim101 pathway was completely dependent on the sensor protein Rim21. Accumulation of Rim20 at the PM was also enhanced by 
tunicamycin treatment in a Rim21-dependent manner (Fig. 5B and C). These results suggest that ER stress causes changes in lipid asymmetry in the PM.

We then hypothesized that the Rim101 pathway is involved in the adaptation to ER stress. To assess this possibility, we investigated the sensitivity of rim21 $\Delta$ and $\Delta$ tether rim21 cells to tunicamycin. As reported previously, $\Delta$ tether cells were hypersensitive to tunicamycin (Fig. 5D) [28]. Cells with a RIM21 deletion were also more sensitive to tunicamycin when compared to WT cells. Moreover, the RIM21 deletion greatly exacerbated the sensitivity of $\Delta$ tether cells to tunicamycin. These results suggest that the Rim101 pathway is at least partially involved in the adaptation to ER stress. Rsb1 and Opt2 are induced by the Rim101 pathway and mediate, directly or indirectly, the flop/transport of sphingoid long-chain bases and phospholipids, respectively [17, 19, 20]. However, neither the deletion of the RBS1 nor OPT2 genes in WT or $\Delta$ tether cells elevated the tunicamycin sensitivity of the resultant mutants (Fig. 5E), indicating that Rim101 pathway dependent-proteins other than Rsb1 and Opt2 play an important role in the adaptation to ER stress.

\section{Impaired complex sphingolipid synthesis activates the Rim101 pathway}

Recently, it was reported that synthesis of sphingolipids, one of the major lipids in the PM, was reduced in $\Delta$ tether cells [35]. Sphingolipids are mostly confined to the outer leaflet of the PM, and thus contribute to the generation of lipid asymmetry. Therefore, we next investigated whether impairment of sphingolipid synthesis activates the Rim101 pathway. Complex sphingolipid synthesis was inhibited by treating cells with 
aureobasidin A, a potent inhibitor of inositol phosphorylceramide synthase, which catalyzes the attachment of phosphoinositol to a ceramide backbone. The activation of the Rim101 pathway was monitored. Aureobasidin A treatment enhanced both the processing of Rim101 and the accumulation of Rim20 at the PM in Rim21-dependent manner (Fig. 6), suggesting that impaired sphingolipid synthesis causes changes in PM lipid asymmetry and activates Rim21. 


\section{DISCUSSION}

In the present study, we found that in $\Delta$ tether cells, which have disrupted ER-PM contacts, the Rim101 pathway was activated even under normal conditions (Fig. 3). Since ER-PM contact sites are the sites of lipid exchange between the cytosolic leaflet of the ER membrane and the inner leaflet of the PM, we speculate that loss of ER-PM contact sites causes a change in the lipid composition of the inner leaflet of the PM, resulting in altered lipid asymmetry and activation of the Rim101 pathway. However, we cannot exclude the possibility that ER-PM contact is serving as a brake for the Rim101 pathway.

In the PM, PtdSer, phosphatidylethanolamine, and phosphatidylinositol are confined to the inner leaflet. The lipid transfer proteins, Osh6 and Osh7, transport PtdSer from the ER to the PM via ER-PM contact sites [34]. We found that Rim101 pathway was not activated in osh6 $\Delta$ osh7 $\Delta$ cells (Fig. 4B). Therefore, the loss of PtdSer transport from the ER to the PM by Osh6 and Osh7 was not sufficient to induce changes in lipid asymmetry to an extent detectable by the Rim101 pathway.

Upon activation of the Rim101 pathway, the Rim101 signaling complex, including Rim20 and an ESCRT III component Snf7, is formed at the PM in a patchy manner [23-25], which resembles the pattern of Rim21 puncta [21]. In yeast two-hybrid assays, Rim21 binds to the arrestin-related protein Rim8, and Rim8 in turn binds to the ESCRT I subunit Vps23 [24]. Therefore, it is likely that the signaling complex is formed at the site where Rim21 is located. In the present research, we revealed that the Rim101 signaling complex mostly formed outside the ER-PM contact sites (Fig. 1). We 
speculate that the Rim101 signaling complex is too large to be accommodated in the narrow space of ER-PM contact sites. However, currently, the local microenvironment of the PM where Rim21 puncta are formed remains unknown. Rim21 is not localized to the eisosome [21], a specialized PM subdomain where scaffold proteins, such as Pil1 and Lsp1, and some transmembrane proteins are enriched [36]. Elucidation of the precise sites of Rim101 signaling on the PM is an important future problem to be solved.

It is noteworthy that the activation of the Rim101 pathway in $\Delta$ tether cells and in tunicamycin-treated WT cells was completely dependent on the sensor protein Rim21. These results suggest that some alterations in the lipid asymmetry of PM are invoked by ER stress and sensed by Rim21, rather than ER stress activating the Rim101 pathway aberrantly without stimulating Rim21. It is known that proteins involved in vesicular trafficking pathways, including secretory pathways, are induced by the unfolded protein response, which is proposed to be an adaptation process to regulate the amount or concentration of unfolded proteins in the ER [37, 38]. Since secretory vesicles, which are composed of lipid bilayers, finally fuse with the PM, some changes in lipid asymmetry may be induced. Alternatively, since the ER is the site of lipid synthesis, it is possible that ER stress affects the production of certain lipid classes, leading to altered lipid asymmetry. It was recently reported that sphingolipid synthesis is altered in $\Delta$ tether cells [35]. Sphingolipids are mostly confined to the extracellular leaflet of the PM lipid bilayer, and thus contribute to generation of asymmetry in the membrane. In the present work, we indeed found that impaired sphingolipid synthesis activated the Rim101 
pathway via the sensor protein Rim21 (Fig. 6). Therefore, an alteration in sphingolipid synthesis together with enhanced vesicular trafficking could cause changes in lipid asymmetry in the PM, followed by activation of the Rim101 pathway (Fig. 7).

ER-PM contact was required for alkaline tolerance in a manner independent of the Rim101 pathway, at least partly (Fig. 3F). Since ER-PM contact is involved in various events, such as phosphatidylinositol 4-phosphate signaling, ion transport, and lipid exchange [30], the loss of ER-PM contact likely causes pleiotropic effects. At present, how ER-PM contact is involved in alkaline tolerance is unknown, and this will be an important issue for future studies.

Cells defective in activation of the Rim101 pathway were more sensitive to the ER stress induced by tunicamycin (Fig. 5D and E). This result indicates that the Rim101 pathway is important for the adaptation to ER stress. As discussed above, ER stress likely causes changes in lipid asymmetry. This altered lipid asymmetry by ER stress must be sensed and be compensated for as maintenance of lipid asymmetry is essential for cell viability [39]. Various genes are induced by the activation of the Rim101 pathway, probably in cooperation with other signaling pathways $[4,6,7,19]$. Both Rsb1 and Opt2 are induced by the Rim101 pathway and are involved directly or indirectly in flop/transport of sphingoid long-chain bases and phospholipids, respectively [18, 19]. However, these proteins do not appear to be critical factors for the adaptation to ER stress (Fig. 5E). Future studies should identify the key proteins that are induced by ER stress and play pivotal roles in the Rim101 pathway-mediated adaptation process. 


\section{AUTHOR CONTRIBUTION}

Keisuke Obara designed the research, performed the experiments, analyzed the data, and wrote the manuscript. Akio Kihara analyzed the data and wrote the manuscript.

\section{ACKNOWLEDGEMENTS}

We are grateful to Dr. C. J. Stefan (Medical Research Council, UK) for providing the $\Delta$ tether strain, to Dr. T. Maeda (University of Tokyo, Japan) for providing the HA-Rim101 plasmid (pFI1), and Dr. T. Yoko-o (National Institute of Advanced Industrial Science and Technology, Japan) for providing the plasmid (pMO13) to visualize the ER.

\section{DECLARATIONS OF INTEREST}

The authors declare no competing interests.

\section{FUNDING}

This work was supported by a Grant-in-Aid for Scientific Research (C) (25440038) to KO and a Grant-in-Aid for Challenging Exploratory Research (25650059) to AK from the Japan Society for the Promotion of Science (JSPS). This work was also supported by Tomizawa Jun-ichi \& Keiko Fund of Molecular Biology Society of Japan for Young Scientist from The Molecular Biology Society of Japan (MBSJ) and by a grant from the Institute for Fermentation, Osaka (IFO) to KO. 


\section{REFERENCES}

1 Peñalva, M. A. and Arst, H. N., Jr. (2002) Regulation of gene expression by ambient $\mathrm{pH}$ in filamentous fungi and yeasts. Microbiol. Mol. Biol. Rev. 66, 426-446

2 Davis, D. A. (2009) How human pathogenic fungi sense and adapt to $\mathrm{pH}$ : the link to virulence. Curr. Opin. Microbiol. 12, 365-370

3 Selvig, K. and Alspaugh, J. A. (2011) pH response pathways in fungi: adapting to host-derived and environmental signals. Mycobiology 39, 249-256

4 Causton, H. C., Ren, B., Koh, S. S., Harbison, C. T., Kanin, E., Jennings, E. G., Lee, T. I., True, H. L., Lander, E. S. and Young, R. A. (2001) Remodeling of yeast genome expression in response to environmental changes. Mol. Biol. Cell. 12, 323-337

5 Lamb, T. M. and Mitchell, A. P. (2003) The transcription factor Rim101p governs ion tolerance and cell differentiation by direct repression of the regulatory genes NRG1 and SMP1 in Saccharomyces cerevisiae. Mol. Cell. Biol. 23, 677-686

6 Lamb, T. M., Xu, W., Diamond, A. and Mitchell, A. P. (2001) Alkaline response genes of Saccharomyces cerevisiae and their relationship to the RIM101 pathway. J. Biol. Chem. 276, 1850-1856

7 Serrano, R., Ruiz, A., Bernal, D., Chambers, J. R. and Arino, J. (2002) The transcriptional response to alkaline $\mathrm{pH}$ in Saccharomyces cerevisiae: evidence for calcium-mediated signalling. Mol. Microbiol. 46, 1319-1333

8 Devaux, P. F. (1991) Static and dynamic lipid asymmetry in cell membranes. 
Biochemistry 30, 1163-1173

9 Verkleij, A. J. and Post, J. A. (2000) Membrane phospholipid asymmetry and signal transduction. J. Membr. Biol. 178, 1-10

10 Gurtovenko, A. A. and Vattulainen, I. (2008) Membrane potential and electrostatics of phospholipid bilayers with asymmetric transmembrane distribution of anionic lipids. J. Phys. Chem. B. 112, 4629-4634

11 Toti, F., Satta, N., Fressinaud, E., Meyer, D. and Freyssinet, J. M. (1996) Scott syndrome, characterized by impaired transmembrane migration of procoagulant phosphatidylserine and hemorrhagic complications, is an inherited disorder. Blood 87, $1409-1415$

12 Fadok, V. A., Voelker, D. R., Campbell, P. A., Cohen, J. J., Bratton, D. L. and Henson, P. M. (1992) Exposure of phosphatidylserine on the surface of apoptotic lymphocytes triggers specific recognition and removal by macrophages. J. Immunol. 148, 2207-2216

13 Emoto, K. and Umeda, M. (2000) An essential role for a membrane lipid in cytokinesis. Regulation of contractile ring disassembly by redistribution of phosphatidylethanolamine. J. Cell Biol. 149, 1215-1224

14 Chen, C. Y., Ingram, M. F., Rosal, P. H. and Graham, T. R. (1999) Role for Drs2p, a P-type ATPase and potential aminophospholipid translocase, in yeast late Golgi function. J. Cell Biol. 147, 1223-1236

15 Furuta, N., Fujimura-Kamada, K., Saito, K., Yamamoto, T. and Tanaka, K. (2007) Endocytic recycling in yeast is regulated by putative phospholipid translocases and 
the Ypt31p/32p-Rcy1p pathway. Mol. Biol. Cell. 18, 295-312

16 Saito, K., Fujimura-Kamada, K., Hanamatsu, H., Kato, U., Umeda, M., Kozminski, K. G. and Tanaka, K. (2007) Transbilayer phospholipid flipping regulates Cdc42p signaling during polarized cell growth via Rga GTPase-activating proteins. Dev. Cell. 13, 743-751

17 Ikeda, M., Kihara, A., Denpoh, A. and Igarashi, Y. (2008) The Rim101 pathway is involved in Rsb1 expression induced by altered lipid asymmetry. Mol. Biol. Cell 19, $1922-1931$

18 Kihara, A. and Igarashi, Y. (2002) Identification and characterization of a Saccharomyces cerevisiae gene, RSB1, involved in sphingoid long-chain base release. J. Biol. Chem. 277, 30048-30054

19 Yamauchi, S., Obara, K., Uchibori, K., Kamimura, A., Azumi, K. and Kihara, A. (2015) Opt2 mediates the exposure of phospholipids during cellular adaptation to altered lipid asymmetry. J. Cell Sci. 128, 61-69

20 Kihara, A. and Igarashi, Y. (2004) Cross talk between sphingolipids and glycerophospholipids in the establishment of plasma membrane asymmetry. Mol. Biol. Cell 15, 4949-4959

21 Obara, K., Yamamoto, H. and Kihara, A. (2012) Membrane protein Rim21 plays a central role in sensing ambient $\mathrm{pH}$ in Saccharomyces cerevisiae. J. Biol. Chem. 287, 38473-38481

22 Nishino, K., Obara, K. and Kihara, A. (2015) The C-terminal cytosolic region of Rim21 senses alterations in plasma membrane lipid composition: insights into 
sensing mechanisms for plasma membrane lipid asymmetry. J. Biol. Chem. 290, 30797-30805

23 Galindo, A., Calcagno-Pizarelli, A. M., Arst, H. N., Jr. and Peñalva, M. A. (2012) An ordered pathway for the assembly of fungal ESCRT-containing ambient $\mathrm{pH}$ signalling complexes at the plasma membrane. J. Cell Sci. 125, 1784-1795

24 Herrador, A., Herranz, S., Lara, D. and Vincent, O. (2010) Recruitment of the ESCRT machinery to a putative seven-transmembrane-domain receptor is mediated by an arrestin-related protein. Mol. Cell. Biol. 30, 897-907

25 Obara, K. and Kihara, A. (2014) Signaling events of the Rim101 pathway occur at the plasma membrane in a ubiquitination-dependent manner. Mol. Cell. Biol. 34, $3525-3534$

26 Pichler, H., Gaigg, B., Hrastnik, C., Achleitner, G., Kohlwein, S. D., Zellnig, G., Perktold, A. and Daum, G. (2001) A subfraction of the yeast endoplasmic reticulum associates with the plasma membrane and has a high capacity to synthesize lipids. Eur. J. Biochem. 268, 2351-2361

27 West, M., Zurek, N., Hoenger, A. and Voeltz, G. K. (2011) A 3D analysis of yeast ER structure reveals how ER domains are organized by membrane curvature. J. Cell Biol. 193, 333-346

28 Manford, A. G., Stefan, C. J., Yuan, H. L., Macgurn, J. A. and Emr, S. D. (2012) ER-to-plasma membrane tethering proteins regulate cell signaling and ER morphology. Dev. Cell 23, 1129-1140

29 Stefan, C. J., Manford, A. G., Baird, D., Yamada-Hanff, J., Mao, Y. and Emr, S. D. 
(2011) Osh proteins regulate phosphoinositide metabolism at ER-plasma membrane contact sites. Cell 144, 389-401

30 Stefan, C. J., Manford, A. G. and Emr, S. D. (2013) ER-PM connections: sites of information transfer and inter-organelle communication. Curr. Opin. Cell Biol. 25, 434-442

31 Longtine, M. S., McKenzie, A., 3rd, Demarini, D. J., Shah, N. G., Wach, A., Brachat, A., Philippsen, P. and Pringle, J. R. (1998) Additional modules for versatile and economical PCR-based gene deletion and modification in Saccharomyces cerevisiae. Yeast 14, 953-961

32 Obara, K., Noda, T., Niimi, K. and Ohsumi, Y. (2008) Transport of phosphatidylinositol 3-phosphate into the vacuole via autophagic membranes in Saccharomyces cerevisiae. Genes Cells 13, 537-547

33 Yeung, T., Gilbert, G. E., Shi, J., Silvius, J., Kapus, A. and Grinstein, S. (2008) Membrane phosphatidylserine regulates surface charge and protein localization. Science 319, 210-213

34 Maeda, K., Anand, K., Chiapparino, A., Kumar, A., Poletto, M., Kaksonen, M. and Gavin, A. C. (2013) Interactome map uncovers phosphatidylserine transport by oxysterol-binding proteins. Nature 501, 257-261

35 Omnus, D. J., Manford, A. G., Bader, J. M., Emr, S. D. and Stefan, C. J. (2016) PI kinase signaling controls ER-PM crosstalk. Mol. Biol. Cell 27, 1170-1180

36 Douglas, L. M. and Konopka, J. B. (2014) Fungal membrane organization: the eisosome concept. Annu. Rev. Microbiol. 68, 377-393 
37 Travers, K. J., Patil, C. K., Wodicka, L., Lockhart, D. J., Weissman, J. S. and Walter, P. (2000) Functional and genomic analyses reveal an essential coordination between the unfolded protein response and ER-associated degradation. Cell 101, 249-258

38 Mori, K. (2009) Signalling pathways in the unfolded protein response: development from yeast to mammals. J. Biochem. 146, 743-750

39 Hua, Z. L., Fatheddin, P. and Graham, T. R. (2002) An essential subfamily of Drs2p-related P-type ATPases is required for protein trafficking between Golgi complex and endosomal/vacuolar system. Mol. Biol. Cell 13, 3162-3177

40 Brachmann, C. B., Davies, A., Cost, G. J., Caputo, E., Li, J., Hieter, P. and Boeke, J. D. (1998) Designer deletion strains derived from Saccharomyces cerevisiae S288C: a useful set of strains and plasmids for PCR-mediated gene disruption and other applications. Yeast. 14, 115-132

41 Winzeler, E. A., Shoemaker, D. D., Astromoff, A., Liang, H., Anderson, K., Andre, B., Bangham, R., Benito, R., Boeke, J. D., Bussey, H., Chu, A. M., Connelly, C., Davis, K., Dietrich, F., Dow, S. W., El Bakkoury, M., Foury, F., Friend, S. H., Gentalen, E., Giaever, G., Hegemann, J. H., Jones, T., Laub, M., Liao, H., Liebundguth, N., Lockhart, D. J., Lucau-Danila, A., Lussier, M., M'Rabet, N., Menard, P., Mittmann, M., Pai, C., Rebischung, C., Revuelta, J. L., Riles, L., Roberts, C. J., Ross-MacDonald, P., Scherens, B., Snyder, M., Sookhai-Mahadeo, S., Storms, R. K., Veronneau, S., Voet, M., Volckaert, G., Ward, T. R., Wysocki, R., Yen, G. S., Yu, K., Zimmermann, K., Philippsen, P., Johnston, M. and Davis, R. W. 
(1999) Functional characterization of the S. cerevisiae genome by gene deletion and parallel analysis. Science 285, 901-906

42 Robinson, J. S., Klionsky, D. J., Banta, L. M. and Emr, S. D. (1988) Protein sorting in Saccharomyces cerevisiae: isolation of mutants defective in the delivery and processing of multiple vacuolar hydrolases. Mol. Cell. Biol. 8, 4936-4948 


\section{Figure Legends}

Fig. 1 Rim101 signaling proceeds at the PM where cortical ER is not associated.

(A and B) YOK3248 (RIM20-GFP) and YOK3250 (SNF7-mRFP) cells were grown to log phase and observed by fluorescence microscopy before and 90 min after alkaline treatment. (A) Arrowheads indicate puncta of Rim20 or Snf7 at the PM. Bar, $5 \mu \mathrm{m}$. (B) The percentages of cells containing Rim20 or Snf7 puncta at the PM are presented as the means \pm S.D. from three independent experiments (**, $P<0.01$; Student's t-test). (C-F) YOK3614 (RIM20-GFP TCB1-mCherry; C and E), YOK3599 (RIM20-GFP SEC61-mCherry; D and E), and YOK3626 (TCB1-GFP SNF7-mRFP vps27A; F) cells were grown to log phase, exposed to an alkaline medium ( $\mathrm{pH}$ 8.0) for $90 \mathrm{~min}$, and visualized by fluorescence microscopy. Micrographs of the middle and peripheral regions of the cells were taken. Arrowheads indicate Rim101 signaling complexes formed at the PM where ER was not associated. Bar, $5 \mu \mathrm{m}$. (E) Rim20-GFP puncta merged at the PM with Tcb1-mCherry or Sec61-mCherry signals were counted and are expressed as percentages. Values represent the means \pm S.D. from three independent experiments.

Fig. 2 ER-PM contact is not essential for formation of Rim101 signaling complex at the PM.

(A) SEY6210 (WT) and ANDY198 (Atether) cells harboring the pMO13 (KAR2 (1-135)-GFP-HDEL; ER marker) plasmid were grown to log phase and visualized by fluorescence microscopy before and 90 min after alkaline treatment. Dashed lines 
indicate the outline of the cells. Bar, $5 \mu \mathrm{m}$. (B) YOK3248 (RIM20-GFP), YOK3249 (RIM20-GFP rim21D), YOK3663 (RIM20-GFP $\Delta$ tether), and YOK3664 (RIM20-GFP rim21 $\Delta$ tether) cells were grown to log phase, exposed to an alkaline medium (pH 8.0) for 90 min, and visualized by fluorescence microscopy. Bar, $5 \mu \mathrm{m}$. (C and D) YOK3248 (RIM20-GFP) and YOK3663 (RIM20-GFP $\Delta$ tether) cells were processed as in (B), and fluorescence intensities associated with (C) whole cells and (D) puncta were measured using ImageJ software (https://imagej.nih.gov/ij/). At least 100 cells or 100 puncta were measured, and the means \pm S.D. are presented. a.u., arbitrary unit; n.s., not significant. (E and F) YOK3250 (SNF7-mRFP) and YOK3643 (SNF7-mRFP $\Delta$ tether) cells were grown to log phase, exposed to an alkaline medium ( $\mathrm{pH}$ 8.0) for $90 \mathrm{~min}$, and (E) visualized by fluorescence microscopy. Arrowheads indicate Snf7 signals at the PM. Bar, $5 \mu \mathrm{m}$. (F) Whole fluorescence intensities of cells were measured using ImageJ software. Values represent the means \pm S.D. from at least 100 cells $\left(^{*}, P<0.05\right.$; Student's t-test).

Fig. 3 The Rim101 pathway is constitutively activated in the $\Delta$ tether mutant.

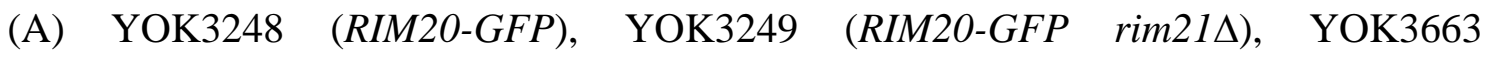
(RIM20-GFP $\Delta$ tether), and YOK3664 (RIM20-GFP rim21s $\Delta$ tether) cells were grown to log phase and visualized by fluorescence microscopy. Bar, $5 \mu \mathrm{m}$. (B) Cells containing Rim20 puncta at the PM in (A) were counted and the percentages are presented as the means \pm S.D. from three independent experiments (*, $P<0.05$; Student's t-test). (C) YOK3250 (SNF7-mRFP) and YOK3643 (SNF7-mRFP $\Delta$ tether) cells were grown to log 
phase and visualized by fluorescence microscopy. Arrowheads indicate Snf7-mRFP puncta at the PM. The lower panels represent the same images as the upper panels but with a longer exposure to the CCD camera. Bar, $5 \mu \mathrm{m}$. (D) YOK3395 (GFP-RIM21C) and YOK3655 (GFP-RIM21C $\Delta$ tether) cells were grown to log phase and visualized by

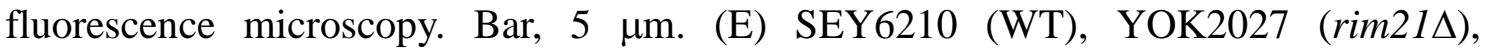
ANDY198 ( $\Delta$ tether), and YOK3657 (rim21s $\Delta$ tether) cells harboring the pFI1 (HA-RIM101) plasmid were grown to log phase and exposed to an alkaline medium (pH 8.0) for $20 \mathrm{~min}$. Total lysates were prepared before and after the alkaline-treatment and immunoblotted with anti-HA antibody. FL and $\Delta \mathrm{C}$ denote the full-length and processed

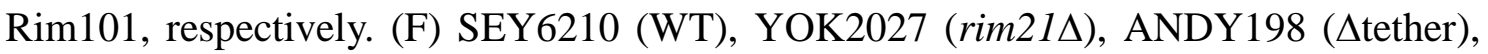
and YOK3657 (rim21 $\Delta \Delta$ tether) cells were grown to a stationary phase, serially diluted at 1:10, spotted on YPD plates or plates at $\mathrm{pH} 8.0$, and grown at $30^{\circ} \mathrm{C}$ for the indicated periods.

Fig. 4 Loss of Osh6/7-mediated PtdSer transfer does not cause activation of the

\section{Rim101 pathway}

(A) SEY6210 (WT) and ANDY198 (Atether) cells harboring the Lact-C2-GFP-p416 (Lact-C2-GFP) plasmid were grown to log phase and visualized by fluorescence microscopy. Arrowheads indicate Lact-C2-GFP in internal membrane structures. Bar, 5 $\mu \mathrm{m}$. In the lower panel, fluorescence intensities of Lact-C2-GFP along the lines indicated in the upper panels were quantified using the Plot Profile function of ImageJ software (https://imagej.nih.gov/ij/). The maximum intensity was set as 1.0 for each line. 


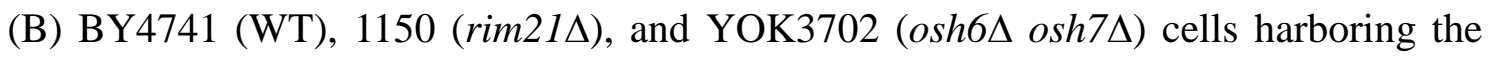
pFI1 (HA-RIM101) plasmid were grown to log phase. Total lysates were prepared and immunoblotted with anti-HA antibody or, to demonstrate uniform protein loading, anti-Pgk1 antibody. FL and $\Delta \mathrm{C}$ denote the full-length and processed Rim101, respectively.

\section{Fig. 5 The Rim101 pathway contributes to adaptation to ER stress.}

(A) SEY6210 (WT) and YOK2027 (rim21A) cells harboring the pFI1 (HA-RIM101) plasmid were grown to log phase and treated with DMSO (control) or $3 \mu \mathrm{g} / \mathrm{mL}$ tunicamycin (Tm) for $60 \mathrm{~min}$. Total lysates were prepared and immunoblotted with anti-HA antibody. FL and $\Delta \mathrm{C}$ denote the full-length and processed Rim101, respectively. (B) YOK3248 (RIM20-GFP) and YOK3249 (RIM20-GFP rim21D) cells were grown to log phase, treated with DMSO (control) or $3 \mu \mathrm{g} / \mathrm{mL}$ tunicamycin (Tm) for $40 \mathrm{~min}$, and visualized by fluorescence microscopy. Bar, $5 \mu \mathrm{m}$. (C) Cells containing Rim20 puncta at the PM in (B) were counted and the percentages are presented as the means \pm S.D. from three independent experiments (*, P < 0.05; Student's t-test). (D) SEY6210 (WT),

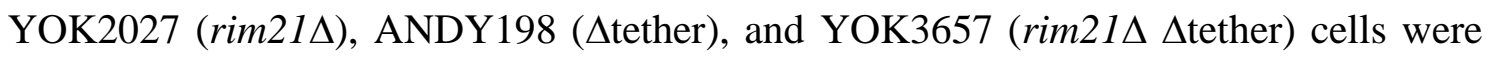
grown to a stationary phase, serially diluted at 1:10, spotted on YPD plates with or without $0.4 \mu \mathrm{g} / \mathrm{mL}$ tunicamycin $(\mathrm{Tm})$, and grown at $30^{\circ} \mathrm{C}$ for the indicated period. (E)

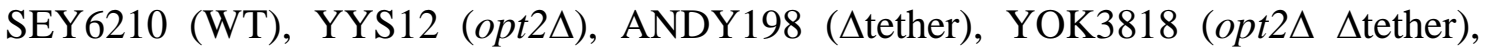

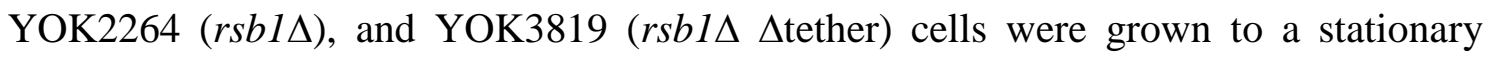


phase, serially diluted at $1: 10$, spotted on YPD plates with or without $0.4 \mu \mathrm{g} / \mathrm{mL}$ tunicamycin $(\mathrm{Tm})$, and grown at $30^{\circ} \mathrm{C}$ for the indicated period.

Fig. 6 The Rim101 pathway is activated by the impairment of complex sphingolipid synthesis.

(A) SEY6210 (WT) and YOK2027 (rim21A) cells harboring the pFI1 (HA-RIM101) plasmid were grown to log phase and treated with ethanol (control) or $2 \mu \mathrm{g} / \mathrm{mL}$ aureobasidin A (AbA) for 60 min. Total lysates were prepared and immunoblotted with anti-HA antibody. FL and $\Delta \mathrm{C}$ denote full-length and processed Rim101, respectively. (B) YOK3248 (RIM20-GFP) and YOK3249 (RIM20-GFP rim21D) cells were grown to log phase, treated with ethanol (control) or $2 \mu \mathrm{g} / \mathrm{mL}$ aureobasidin A (AbA) for $60 \mathrm{~min}$, and visualized by fluorescence microscopy. Bar, $5 \mu \mathrm{m}$. (C) Cells containing Rim20 puncta at the PM in (B) were counted and the percentages are presented as the means \pm S.D. from three independent experiments ( ${ }^{* *}, \mathrm{P}<0.01$; Student's t-test).

Fig. 7 Working hypothesis of the Rim101 pathway-mediated adaptation process in response to ER stress.

ER stress causes alterations in PM lipid asymmetry through changes in sphingolipid synthesis and enhanced vesicular trafficking. Rim21 senses such alterations at the PM and activates the Rim101 pathway for adaptation. 
Table I. Yeast strains used in this study

\begin{tabular}{|c|c|c|}
\hline Strain & Genotype & Source \\
\hline BY4741 & MATa his3 $\Delta 1$ leu $2 \Delta 0$ met $15 \Delta 0$ ura3 $\Delta 0$ & {$[40]$} \\
\hline 1150 & BY4741, rim21 $\Delta:: K a n M X 4$ & [41] \\
\hline SEY6210 & MAT $\alpha$ his3 leu2 ura3 trp1 lys2 suc2 & {$[42]$} \\
\hline SEY6210.1 & MATa his3 leu2 ura3 trp1 lys2 suc2 & {$[28]$} \\
\hline \multirow[t]{2}{*}{ ANDY198 } & 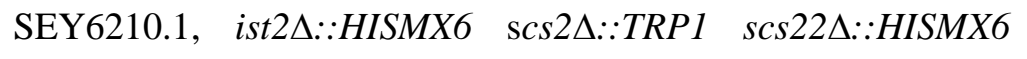 & {$[28]$} \\
\hline & 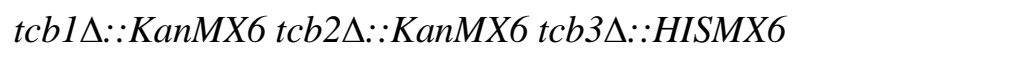 & \\
\hline YOK2027 & SEY6210, rim21 $::$ KanMX4 & {$[21]$} \\
\hline YOK2264 & 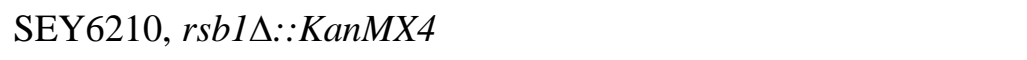 & This study \\
\hline YOK3248 & SEY6210, RIM20-GFP::TRP1 & {$[25]$} \\
\hline YOK3249 & SEY6210, RIM20-GFP::TRP1 rim21ه::KanMX & {$[25]$} \\
\hline YOK3250 & SEY6210, SNF7-mRFP::NatNT2 & {$[25]$} \\
\hline YOK3395 & SEY6210, $\mathrm{P}_{A D H^{-}}$GFP-RIM21C::URA3 & {$[22]$} \\
\hline YOK3599 & SEY6210, RIM20-GFP::TRP1 SEC61-mCherry::KanMX6 & This study \\
\hline YOK3614 & SEY6210, RIM20-GFP::TRP1 TCB1-mCherry::KanMX6 & This study \\
\hline \multirow[t]{2}{*}{ YOK3626 } & SEY6210, $\quad$ TCB1-GFP::TRP1 & This study \\
\hline & vps274::KanMX4 & \\
\hline YOK3643 & ANDY198, SNF7-mRFP::NatNT2 & This study \\
\hline YOK3657 & ANDY198, rim21 $::$ NatNT2 & This study \\
\hline YOK3663 & ANDY198, RIM20-GFP::LEU2 & This study \\
\hline YOK3664 & ANDY198, RIM20-GFP::LEU2 rim21 $:: N a t N T 2$ & This study \\
\hline YOK3655 & ANDY198, $\mathrm{P}_{A D H}-G F P-R I M 21 C:: U R A 3$ & This study \\
\hline
\end{tabular}


YOK3702

BY4741, osh6A::NatNT2 osh7A::HIS3

YOK3818 ANDY198, opt2A::NatNT2

YOK3819 ANDY198, rsb14::URA3

YYS12 SEY6210, opt2A::KanMX4
This study

This study

This study

[19] 
Fig. 1

A

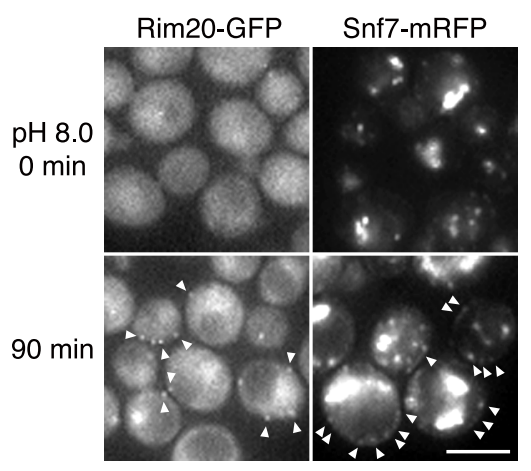

C

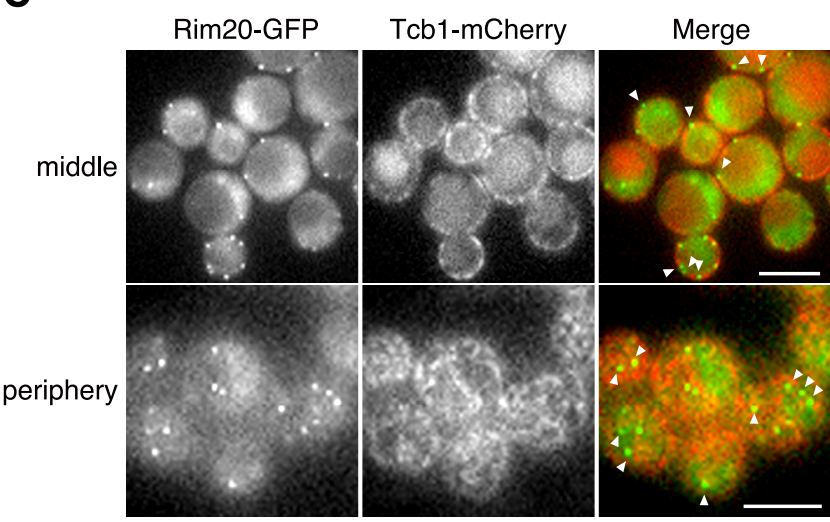

B

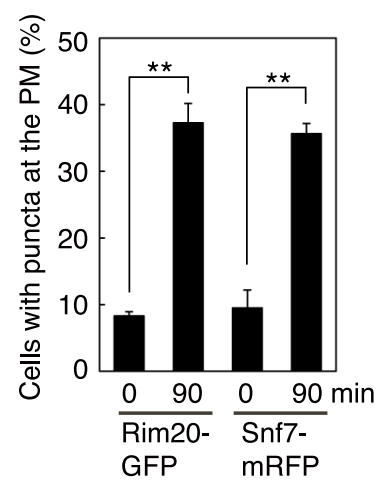

D

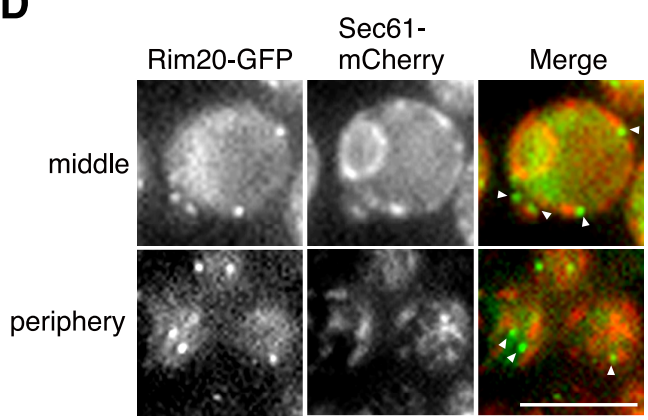

E

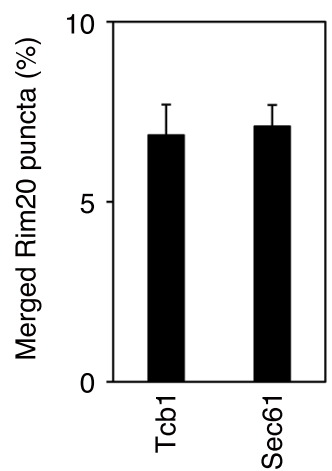

F

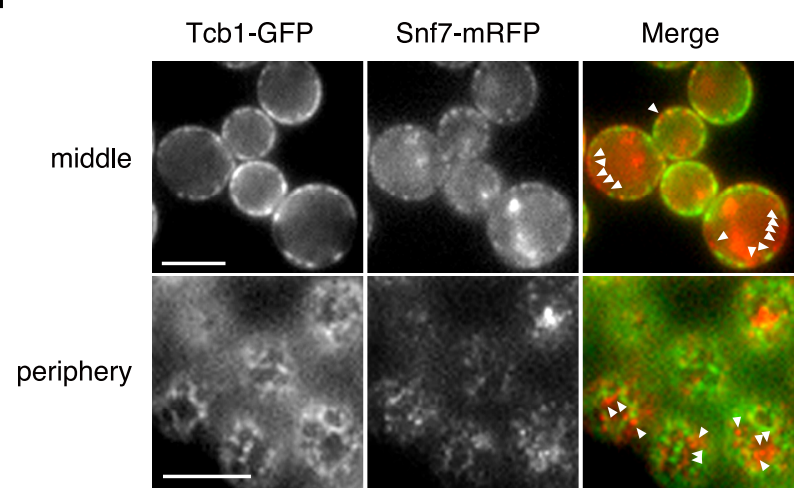


Fig. 2

A

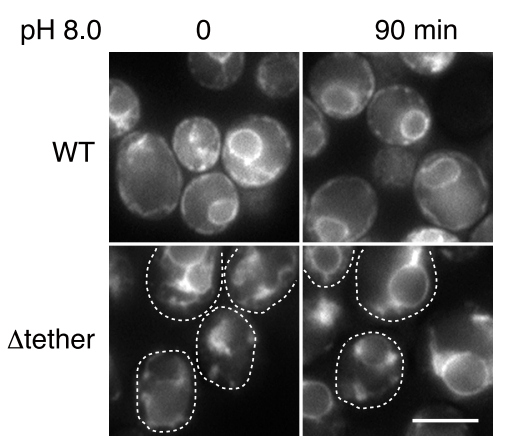

C

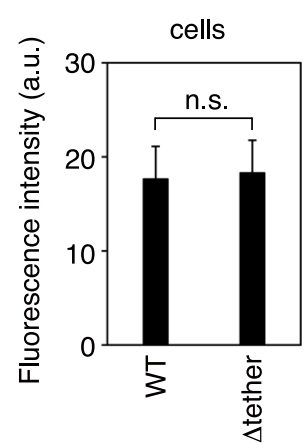

E

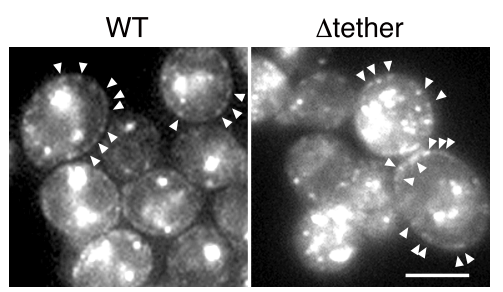

B

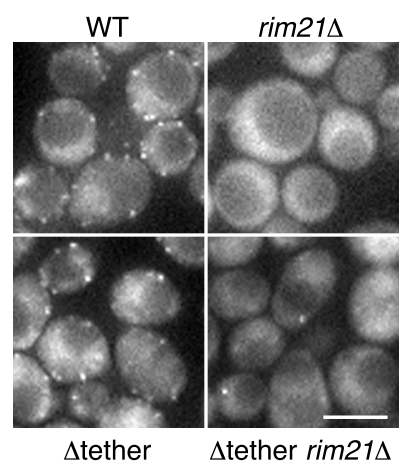

D

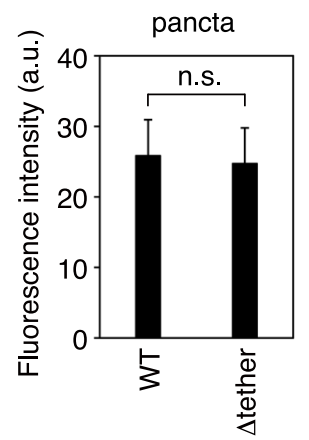

F

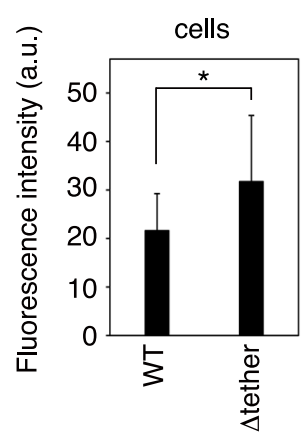


Fig. 3

A

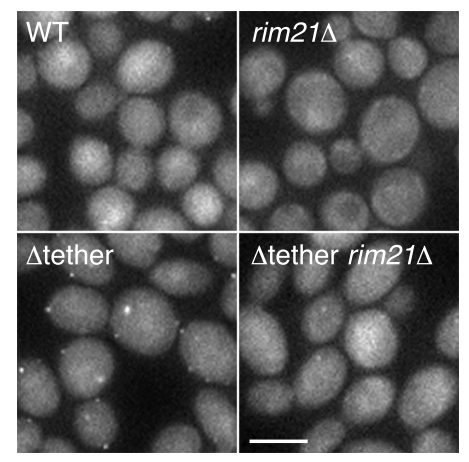

D

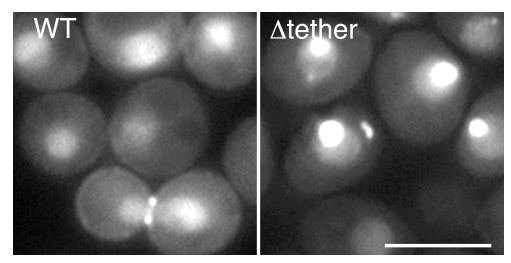

E

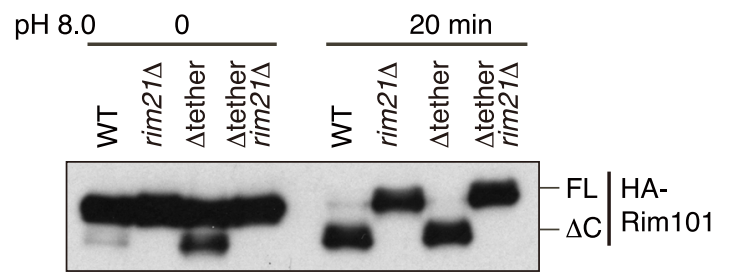

C
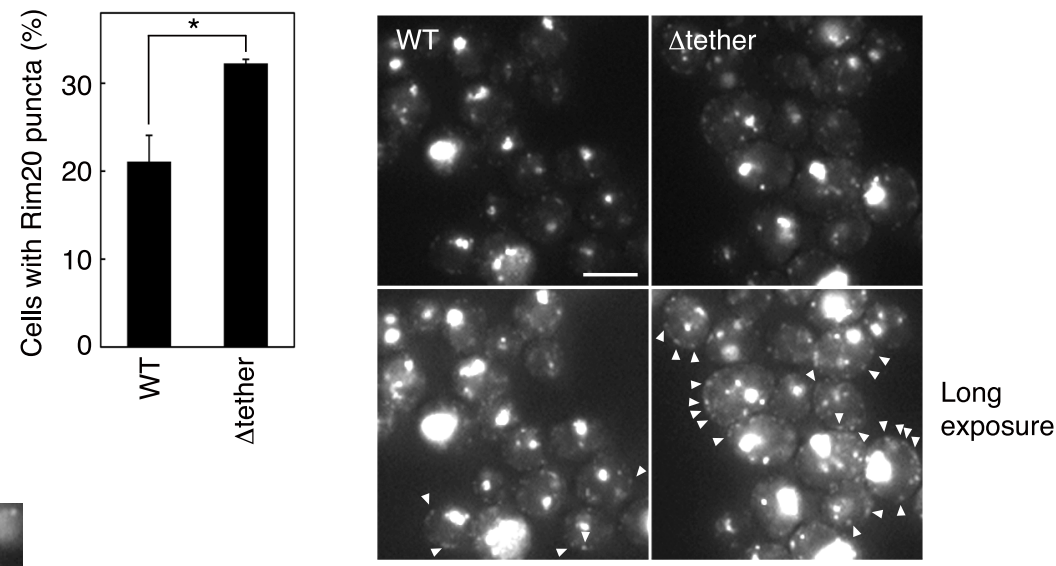

F

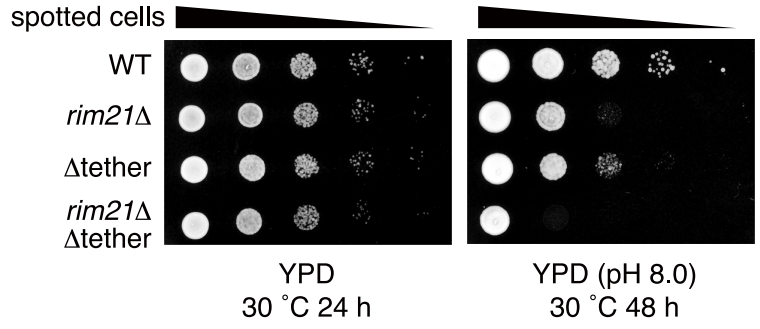


Fig. 4

A
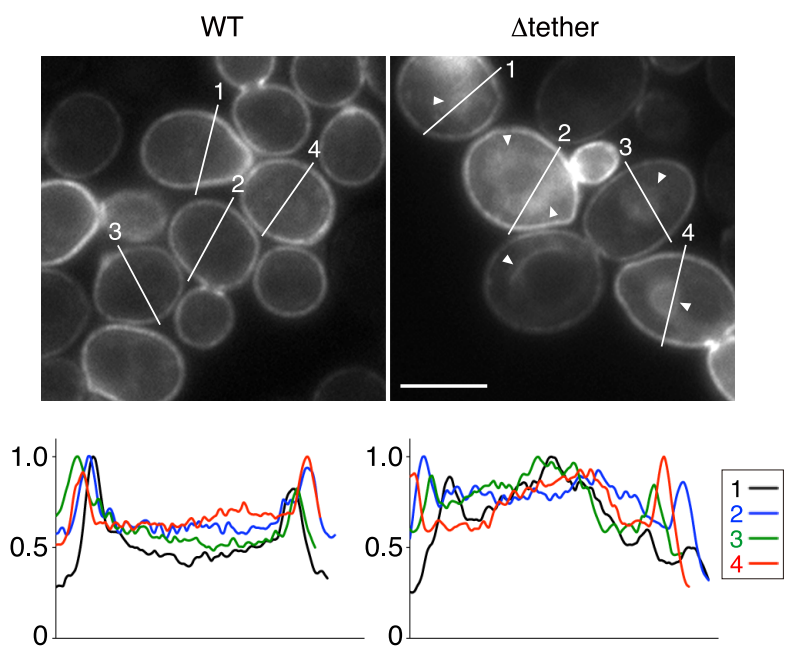

B

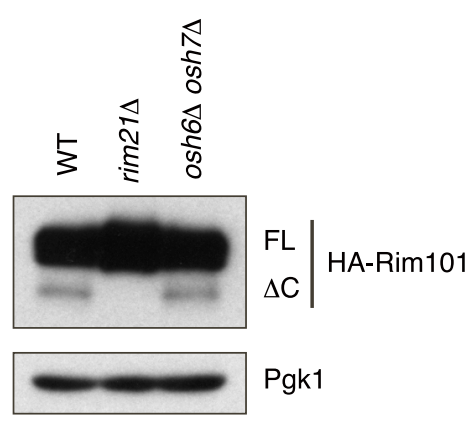


Fig. 5
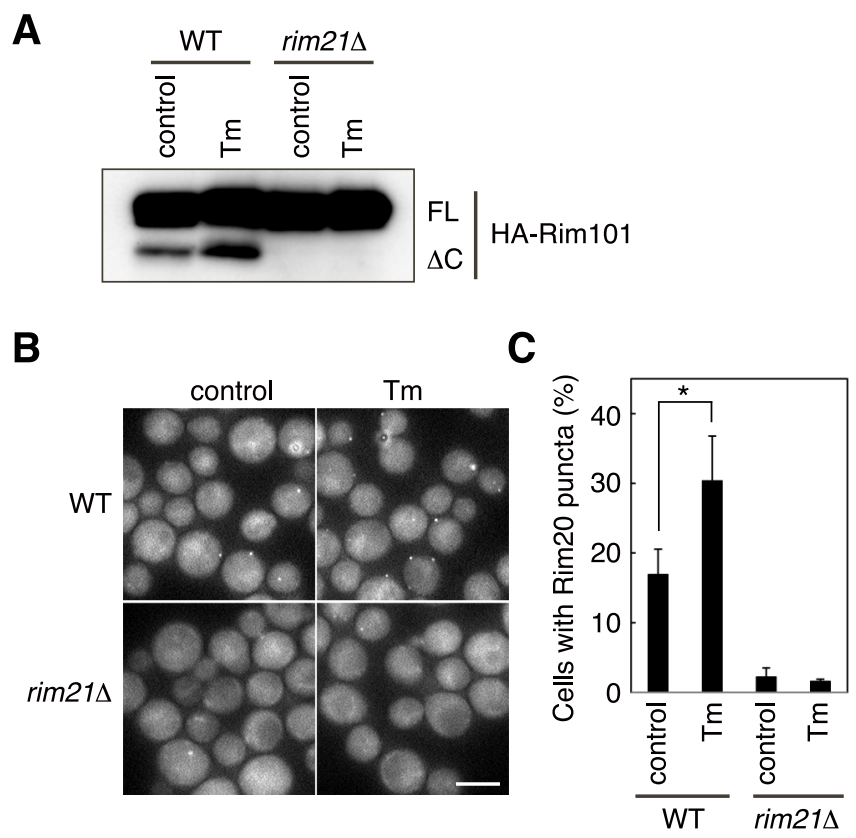

D

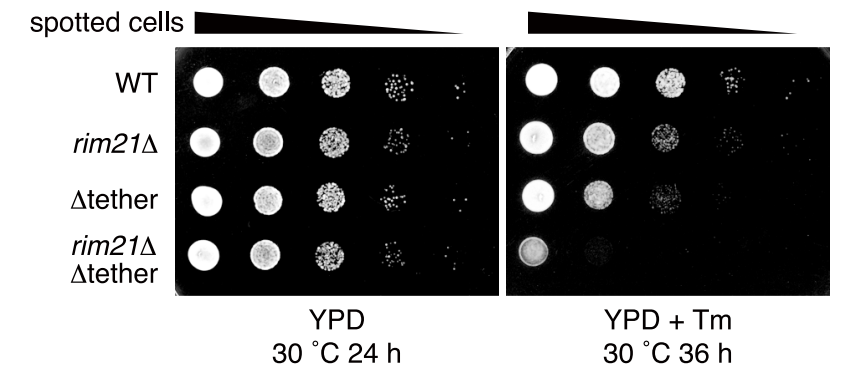

E

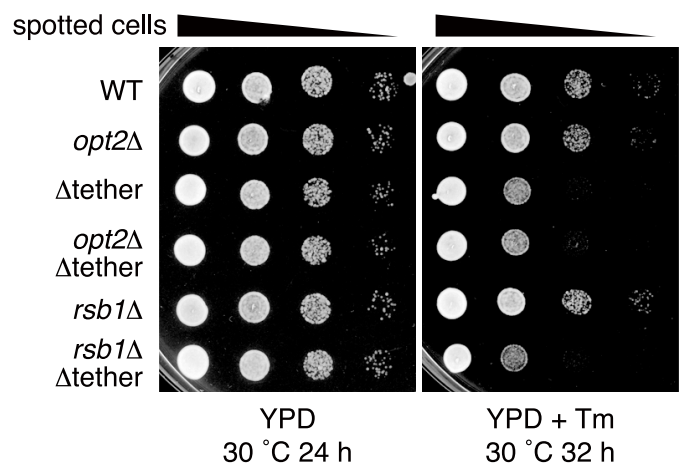


Fig. 6

A

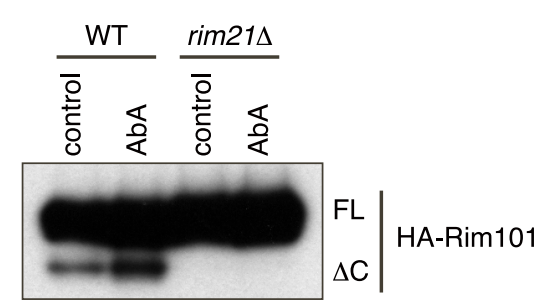

B

C
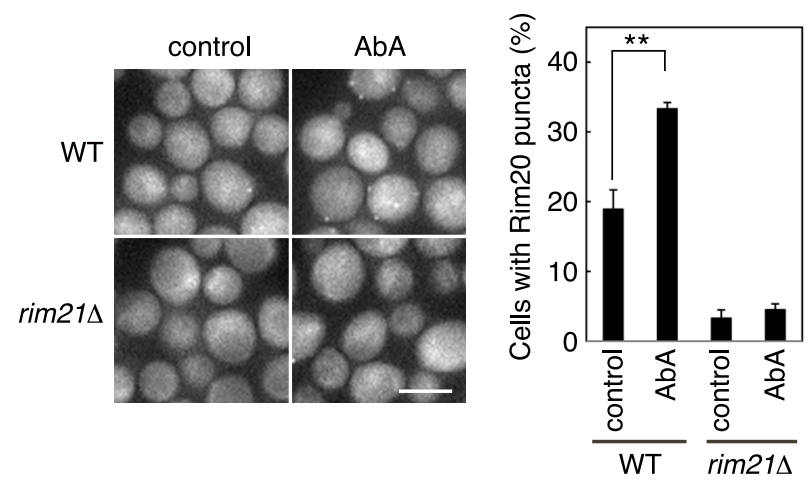
Fig. 7

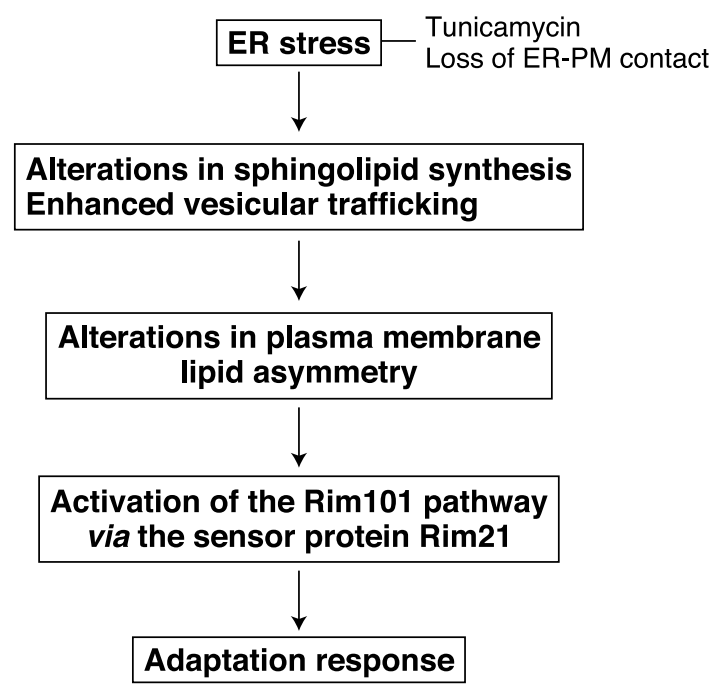

